\title{
Complex event processing for smart grid active management in distributed new energy generation environment
}

\author{
Xiang-Rong $\mathrm{Zu}$ and Yan Bai \\ Control \& Computer Engineering, North China Electric Power University, \\ Changping, Beijing, 102206, China \\ 'E-mail: \{zuxr\&by\}@ncepu.edu.cn \\ www.ncepu.edu.cn \\ Su-Ling Ma \\ Beijing China-Power Information Technology CO., LTD \\ Haidian, Beijing, 100192, China \\ E-mail: masuling@sgitg.sgcc.com.cn
}

The open smart grid reference architecture (SGRA), based upon the GridWise ${ }^{\circledR}$ interoperability framework, has proposed real-time integration architecture, EDA and robust CEP as key enabling information architectural capabilities. EDA complements SOA in event based requirement analysis \& design paradigm. CEP as a promising approach for analyzing various streams of big data, detecting complex event patterns \& relationships, real-time response action, and supporting business decision making in near real-time, can discover business opportunities \& crisis situations for active management. This paper analyzed smart grid context awareness, real-time responses \& decision making needs, difficulties \& challenges, and CEP real-time big data processing capability, summarized smart grid CEP application present achievements and existing challenges; As a case study of active management on active distribution systems (ADSs) in new energy power system, this paper has studied providing CEP capabilities based upon event-driven SOA communication infrastructure for collaboration application, this paper designs a distributed hierarchical CEP system architecture to coordinate control \& active management in ADS; As CEP and EDA can fully separately design and coordination well, and good industry standards support, this architecture has more advantages than MAS design. CEP and ED-SOA provide near real-time services cooperation system, highly wide area awareness $\&$ responses, that can satisfy smart grid core ICT requirement.

Keywords: Smart grid; Complex event processing (CEP); big data; Event-driven SOA; Ontology; Active management

\section{Introduction}

The smart grid revolution demands a huge effort in redesigning and enhancing current power networks, as well as integrating emerging scenarios such as distributed generation, renewable energies or the electric vehicle. As that active 
resources actually change the traditional passive distribution network to be an active one. Active distribution systems (ADSs) are "distribution networks with systems in place to control and manage a combination of distributed energy resources (DERs), defined as generators, loads and storage. Intrinsically, new network solutions and active resources call for novel ICT solutions for network operation and asset management providing intelligence to active networks [1]. Next generation systems such as smart grid will be large, complex, dynamic and heterogeneous. Therefore decentralized and self-organizing mechanisms present promising approaches to deal with the massive amount of data generated by different components in the smart grid. The Smart Grid Reference Architecture (SGRA) proposed based upon the Grid Wise ${ }^{\circledR}$ Interoperability Context-Setting Framework has proposed real-time integration architecture, Event Driven Architecture (EDA) and robust Complex Event Processing (CEP) as key enabling information architectural capabilities to realize decentralized, reactive and self-organizing mechanism [2].

\section{Event Driven and Complex Event Processing for Smart Grid Real- Time Integration Architecture}

Smart grid architecture is to provide real-time decision making which is possible only if data can be harnessed as it is generated and is applied towards a specific objective that requires data as it happens. An event can be considered as any notable condition that happens inside or outside your IT or your business. Exchange of event information among multiple interconnected sub-systems has become imperative for integrated and secure operation of the power system. EDA, an "event oriented" system, which is a new proposed software architecture pattern for promoting the production, detection, consumption, and reaction to events [3] .CEP used to modeling and detecting dynamic situations events justin-time, which facilitates the development of sense-and-respond software capable of processing events extracted from large volumes of real-time data streams.

In complex distributed energy system, observing failures and other desired or undesired system operation behavior patterns is difficult, these systems can generate data in order of gigabytes every day, which makes a challenge to process in the course of predicting upcoming critical problems or identifying relevant behavior patterns. Low level data has to be processed, correlated and synthesized in order to create high level, decision helping data. The actual value of this high level data lays in its availability at the time of decision making in real time. CEP has emerged as a model to respond to high frequency data streams. CEP engines look for patterns and relationships between unrelated events that signal new opportunities or critical threats and trigger a reaction in 
response to the detected pattern. The industry has increasingly adopted Complex Event Processing (CEP) as a solution to fast and efficient processing of these event streams in real-time. CEP focuses on detecting patterns of interest on event streams that are correlated in a causal, spatial or time dimension process, analyzing their impact and acting upon them. In smart grid, the paper [4] designed a CEP-based distributed control framework improving upon existing "smart grid management" frameworks and other complex event processing platforms by providing a robust and persistent communication infrastructure. Papers [5-6] analyzed demand response optimization (DR) is a key component of Smart Grids, and existing DR programs fail to effectively leverage the nearreal time information available from AMIs and BANs to adapt to increasing dynamism in energy use profiles. These papers investigated the use of Semantic Complex Event Processing (CEP) to model and detect dynamic situations in a campus micro grid that facilitates DR by abstracting event streams from various information sources including smart meters, sensors, and online services as a logical stream of events. A SCE Pter Semantic CEP framework has given and illustrated the efficacy of pattern detection for DR by applying them to real time event streams in the USC Campus micro grid. Paper [7] presented an implementation of complex event processor (CEP) for processing high level power system events from the perspective of a multi-area system. Online real time data on the high voltage Indian national grid is used for driving the CEP system with the objective of detecting the occurrence of complex events. The methodology, implementation and results of the proposed CEP system are presented. Paper [8] analyzed the temporal, spatial and electrical correlation between several successive failure events during the initial stage in cascading failure, and gave a hierarchy model and correlation analysis based on CEP technology. A case study shows the proposed method effectiveness. As we presented in this section, CEP engines process events in the operation of large scale distributed smart gird systems to understand systems, detect patterns, find relationship (causality, membership or timing) between events. CEP has good potential for smart grid active management.

\section{Active Management Architecture in Distributed New Energy Generation Environment}

The penetration of distributed generation (DG), especially based on Renewable Energy Sources (RES), will continue due to environmental reasons. One of the main barriers for the penetration of active resources at distribution network level is the complexity of the interconnection process. From the network management point of view the increasing amount of DG is often considered reluctantly as it brings the complexity of a transmission network to distribution network level. 
The main reason for the complexity is caused by the present methods for managing the distribution networks as well as the features of different active resource components themselves, which are not sufficiently developed to enable easy interconnection. The intermittent (non-dispatch able, uncertain and uncontrolled) production connected into the passive network brings the complexity of management [1]. Situational awareness and observability towards the new energy power system operation is more and more important, Sensors, communication systems and physical power networks together forming a large distributed Cyber-Physical Energy System (CPES), reliable and flexible operation needs new information system real time integration architecture. The power system operation will produce large amounts of events, that can be arranged into either an event stream or an event cloud, CEP act as an Event Processing Agents (EPA), actually as a context-based situation observer and manager can intelligently processes them into decision-making knowledge, and trigger action in time. These context-based event processing is the next 'big thing' and should shape the future of the computing, as the most promising paradigm that will extend SOA forming the event-driven smart grid real-time integration architecture.

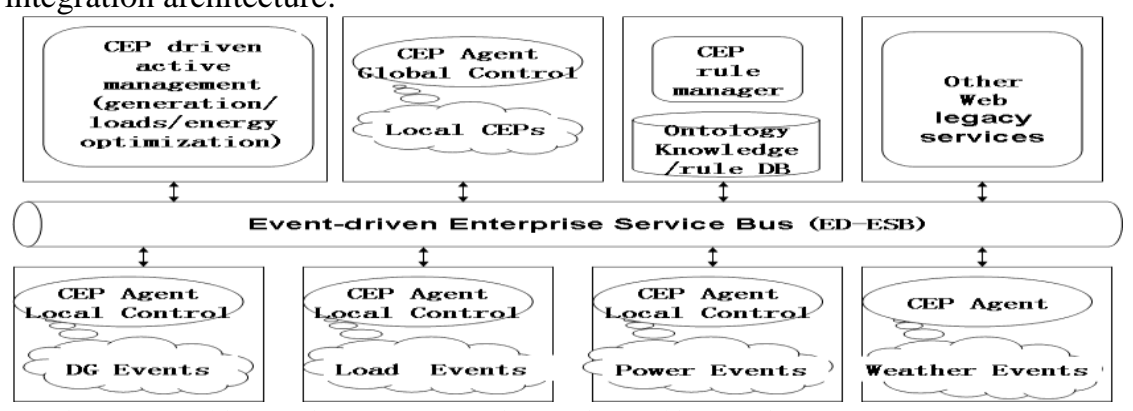

Fig. 1. Event driven active management integration architecture in new energy power system

As shown in Fig.1, an event driven active management integration architecture in new energy power system has given. CEP can be layered deployed, that adhered to the distributed hierarchical coordinated control architecture model in ADSs control design in new energy power system. The platform has coordinated EDA and CEP design to realize real time decisionmaking by means of CEP agents rapidly processing capabilities, implementing power system operation parameters, DG sub-system, local load in regions, weathers and environment related vast data collecting, monitoring and real time analyzing with local computation. CEP can realize local operation optimization management firstly to reduce communication burden, and then coordinate with upper level CEP Agent. CEP Agent can be hierarchically associated, and realize coordination control with the highest level CEP performing global correlation 
and optimal control. CEP can promote situational awareness, system observability, fast response capability and action reactivity.

Event-driven Enterprise Service Bus (ED-ESB) has adopted for information exchange communication platform for CEP and application components. According to custom business rule to realize event driven interconnection and coordination, automating the business workflow process. Every CEP node's event processing and CEP node hierarchical model has given in Fig.2, the configurable CEP rule engine is the core of CEP, under the rule engine driven, CEP performs data aggregations, context awareness, and action planning, CEP agents communicated with real time publish/subscribe model, SOA legacy services can still continue to use the request / response model, to realize the both mode complementary advantages.

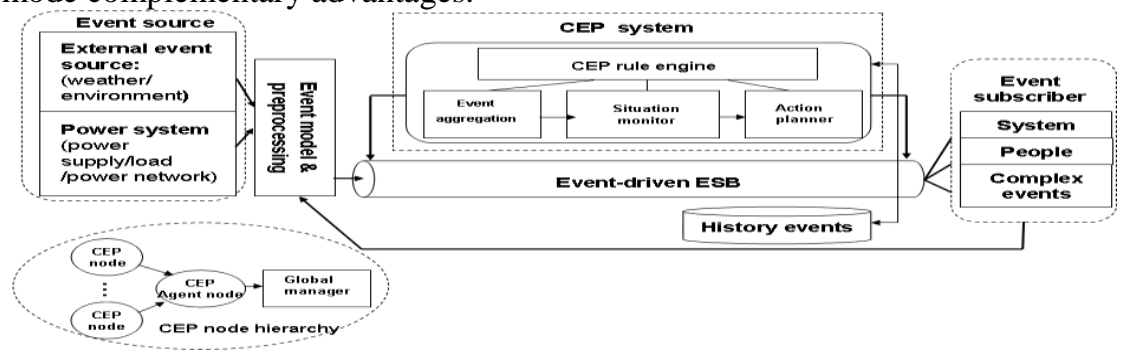

Fig. 2. Event processing flow \& CEP node hierarchical model

The design architecture advantages. Although Multi-Agent System (MAS) as popular active management technology has wildly applied in smart grid, especially in micro-gird, ADSs[9], but MAS has disadvantage for complex communication design, lack of industry standards and support for knowledge base development platform other issues. CEP is lightweight, especially for vast active data processing, as a high performance custom agent, can solve CPES Information physical perception layer specific operation condition monitoring, easy to coordinate with ED-ESB communication platform, that providing standard based event-driven coordination.

\section{Conclusions}

The open smart grid reference architecture (SGRA) has proposed real-time integration architecture for interoperability, EDA and robust CEP as the key enabling information architectural capabilities. This paper analyzed CEP realtime event stream processing capability, summarized smart grid CEP application present achievements and existing challenges; As a case study of active management on ADSs in new energy power system, this paper has studied providing CEP capabilities based upon event-driven SOA communication 
infrastructure for collaboration application, this paper designs a distributed hierarchical CEP system architecture to coordinate control \& active management in ADS; As CEP and EDA can fully separately design and coordination well, and good industry standards support, this architecture has more advantages than MAS design. CEP and ED-SOA provide near real-time services cooperation system, highly wide area awareness \& responses, that can satisfy smart grid core ICT requirement.

\section{References}

1. Taylor J, Jupe S, Celli G, et al. Assessing the impact of ICT on the reliability of active distribution systems[C]. International Conference and Exhibition on Electricity Distribution. IET, 2013:156-161.

2. Giroti T. Integration roadmap for smart grid: from accidental architecture to smart grid architecture [J]. Bridge Energy Goup Inc, 2009: 1-30.

3. Luck ham D C. The power of events: an introduction to complex event processing in distributed enterprise systems [M]. Reading: Addison-Wesley, 2002.

4. Srinivasagopalan S, Mukhopadhyay S, Bharadwaj R. A complex-eventprocessing framework for smart-grid management[C]. Cognitive Methods in Situation Awareness and Decision Support (CogSIMA), 2012 IEEE International Multi-Disciplinary Conference on. IEEE, 2012:272-278.

5. Zhou Q, Simmhan Y, Prasanna V. On using semantic complex event processing for dynamic demand response optimization[R]. Technical report, Computer Science Department, University of Southern California, 2012.

6. Zhou Q, Simmhan Y, Prasanna V. Towards an inexact semantic complex event processing framework[C]. Proceedings of the 5th ACM international conference on Distributed event-based system. ACM, 2011: 401-402.

7. Pradeep Y, Khaparde S A. Complex event processing of high level events in multi-area power grid: An Indian perspective[C].Power and Energy Society General Meeting, 2010 IEEE. IEEE, 2010: 1-6.

8. Xu Qian, Bai Xiaomin, Xu Dechao. Cascading failure diagnosis by application of complex event processing [J].Automation of Electric Power Systems, 2011, 35(3): 5-20 (in Chinese).

9. S. D. J. McArthur; E. M. Davidson; V. M. Catterson; A. L. Dimeas; N. D. Hatziargyriou; F. Ponci; T. Funabashi, Multi-agent systems for power engineering applications-part II: technologies, standards, and tools for building multi-agent systems, IEEE Transactions on Power Systems, Vol. 22, No. 4, November 2007. 